\title{
新型 $\mathrm{GdGa}_{3-x} \mathrm{Mn}_{x}$ 结构和磁性
}

\author{
何强, 刘汉源, 郭永权* \\ 华北电力大学能源动力与机械工程学院, 北京 102206 \\ *联系人, E-mail: yqguo@ncepu.edu.cn \\ 2014-03-05 收稿, 2014-03-25 接受, 2014-05-23 网络版发表 \\ 国家自然科学基金(11274110)资助
}

\begin{abstract}
摘要 应用非自耗真空电弧炉制备稀土 $\mathrm{GdGa}_{3-x} \mathrm{Mn}_{x}(x=0,0.1,0.2,0.3)$ 系列合金. 采用粉末 $\mathrm{X}$ 射线衍射(XRD)研究合金的相结构, 利用晶面指数尝试法及最小二乘法(TREOR)对衍射数 据进行晶面指标化，采用 Rietveld 全谱拟合分析方法测定了 $\mathrm{GdGa}_{3-x} \mathrm{Mn}_{x}$ 的晶体结构. 结果表 明, $\mathrm{GdGa}_{3-x} \mathrm{Mn}_{x}(x=0,0.1,0.2,0.3)$ 主相结晶成六方型结构的合金化合物，空间群 P $8 / \mathrm{mmm} . \mathrm{Gd}$ 原子占据 $1 \mathrm{a}(0,0,0)$ 晶位, $\mathrm{Ga}$ 原子分别占据 $2 \mathrm{~d}(1 / 3,2 / 3,1 / 2)$ 和 $2 \mathrm{e}(0,0, \mathrm{z})$ 晶位, $\mathrm{Mn}$ 原子部分替 代 $\mathrm{Ga}$ 占据 $2 \mathrm{e}$ 晶位. 应用振动样品磁强计(VSM) 测量了 $\mathrm{GdGa}_{3-x} \mathrm{Mn}_{x}$ 样品的磁化曲线 (M-H). 在 Gd-Ga-Mn 体系中, Mn 原子对磁性有贡献, 在低 Mn 区合金呈顺磁性; 随 Mn 含量增加, 呈 现出弱铁磁性和顺磁性的混合. 在 0 1.5 T 磁场下对其 $\mathrm{M}-\mathrm{H}$ 曲线进行拟合, 随着 $\mathrm{Mn}$ 含量的 增加, 磁化率 $\chi_{\mathrm{P}}$ 与饱和磁化强度 $M_{\mathrm{s}}$ 先增后减, 而与外场相关的参数 $a, b, c$ 先减后增, 并且均 在 $x=0.2$ 时达到极值, 说明 $\mathrm{Mn}$ 的掺杂对 $\mathrm{GdGa}_{3-x} \mathrm{Mn}_{x}$ 的磁性能产生了较大影响.
\end{abstract}

关键词

$\mathrm{GdGa}_{3-x} \mathrm{Mn}_{x}$ Rietveld 结构精修 磁性
稀土-半导体元素所组成的 $\mathrm{RX}_{3}(\mathrm{R}$ 为稀土, $\mathrm{X}=\mathrm{Sn}$, $\mathrm{Pb}, \mathrm{In}, \mathrm{Ga})$ 金属化合物大多具有 $\mathrm{AuCu}_{3}$ 型晶体结构, 如 $\mathrm{RPb}_{3}, \mathrm{RSn}_{3}, \mathrm{Rln}_{3}$ 和 $\mathrm{RGa}_{3}$. 在过去的几十年中, 这 些体系由于稀土元素 $4 \mathrm{f}$ 电子的特殊性质而呈现出多 种物理性能，如超导、复杂的磁性、晶体场效应、 Kondo 效应或多轴磁结构等, 引起广泛的关注 ${ }^{[1 \sim 3]}$. 虽然对 R-Ga 化合物结构及物理性能的研究已有 10 余年, 但基本上多属于 $\mathrm{RGa}_{2}$ 型合金化合物. 在 $\mathrm{RGa}_{2}$ 家族中, 除 $\mathrm{TmGa}_{2}$ 外, 其余二元化合物均由 $\mathrm{R}$ 和 $\mathrm{Ga}$ 原子交替排列形成简单六方 $\mathrm{AlB}_{2}$ 型结构 $(P 6 / \mathrm{mmm})^{[4]}$, 其中稀土 $\mathrm{R}$ 原子占 $1 \mathrm{a}(0,0,0)$ 晶位, 而非磁性原子 $\mathrm{Ga}$ 占 $2 \mathrm{~d}(1 / 3,2 / 3,1 / 2)$ 晶位. 据报道 $\mathrm{GdGa}_{2}$ 在 $23.6 \mathrm{~K}$ 时为 反铁磁结构 ${ }^{[5,6]}$, 但是在奈尔温度附近比热未呈现跳 变 ${ }^{[7]}$. 另外, 有报道称低于奈尔温度可观察到磁相转 变, 转变温度 $T_{\mathrm{t}}=5 \mathrm{~K}^{[6]}$. 基于中子散射研究结果, $\mathrm{GdGa}_{2}$ 在温度为 $2 \mathrm{~K}$ 时, 磁结构具有不对称性, 中子 散射向量 $Q \leqslant 0.39,0.39,0>$. 对于 $\mathrm{Gd}(\mathrm{Ga}, \mathrm{Al})_{2}$ 合金,
当 $x \leqslant 1.5$ 时, $\mathrm{GdGa}_{2-x} \mathrm{Al}_{x}$ 和 $\mathrm{GdGa}_{2}$ 具有相同的晶体结 构 ${ }^{[8]}$. 当 $x<0.6$ 时, 点阵常数的比值 $c / a \approx 0.97$; 当 $x>0.75$ 时, $c / a \approx 0.84$. 与此同时, 磁结构具有反铁磁 性 $^{[9,10]}$.

目前对 $\mathrm{RGa}_{3}$ 化合物研究还较少. 在 $\mathrm{RGa}_{3}$ 家族中, 仅发现有 $\mathrm{ErGa}_{3}, \mathrm{TmGa}_{3}$ 和 $\mathrm{LuGa}_{3}$, 它们都具有立方 $\mathrm{AuCu}_{3}$ 型结构, 而其他稀土与 $\mathrm{Ga}$ 都不能形成 $\mathrm{AuCu}_{3}$ 型结构化合物 ${ }^{[11]} . \mathrm{ErGa}_{3}$ 呈现出调幅式反铁磁结构 $a[-\tau,-\tau, 0]$, 传播向量 $\tau=0.020$, 奈尔温度为 $2.8 \mathrm{~K}$, 与 二级相变温度相同. 在 $2 \mathrm{~K}$ 温度下的磁矩为 $6.1 \pm 0.3 \mu_{\mathrm{B}}$. 但由于 $\mathrm{Er}^{3+}$ 离子的特殊性质, 使得调幅结构在低温区 有可能被第三类反铁磁结构代替, 形成复杂的磁结 构. 因而与其相邻的 $\mathrm{TmGa}_{3}$ 引起了人们的兴趣. $\mathrm{TmGa}_{3}$ 具有 2 个一级相变, 相变温度很近, 分别为奈 尔温度 $T_{\mathrm{N}}=4.26 \mathrm{~K}$ 和四极反铁磁有序温度 $T_{\mathrm{Q}}=4.29 \mathrm{~K}$, 低于 $T_{\mathrm{N}}$ 温度区, 磁性呈现多轴各向异性, $2 \mathrm{~K}$ 温度下 的磁矩为 $2.3 \mu_{\mathrm{B}}$. 在稀土与半导体元素形成的 $1: 3$ 型

引用格式: 何强, 刘汉源, 郭永权. 新型 $\mathrm{GdGa}_{3-\mathrm{x}} \mathrm{Mn}_{x}$ 结构和磁性. 科学通报, 2014, 59: 1769-1775 He Q, Liu H Y, Guo Y Q. Structure and magnetic properties of novel GdGa ${ }_{-x} \mathrm{Mn}_{x}$ intermetallic compounds (in Chinese). Chin Sci Bull (Chin Ver), 2014, 59: 1769-1775, doi: 10.1360/N972013-00033 
化合物中, 稀土 $\mathrm{R}$ 与半导体元素的原子半径比值在 1.4 附近: $\mathrm{ErGa}_{3}$ 为 $1.406, \mathrm{TmGa}_{3}$ 为 $1.431, \mathrm{LuGa}_{3}$ 为 1.398. 这说明稀土镓基 $1: 3$ 型化合物的形成和稳定 性与原子半径比、电负性差和价态密切相关 ${ }^{[12]}$. 单纯 从几何因素考虑, 为了形成 $\mathrm{RGa}_{3}$ 化合物, 稳定元素 的原子尺寸须满足这种半径比值, 才能形成稳定的 $\mathrm{R}(\mathrm{M}, \mathrm{Ga})_{3}$ 化合物. 因此，通过掺杂少量的 $3 \mathrm{~d}$ 过渡族 元素, 可以形成稳定的伪二元的 $R(M, G a)_{3}$ 型化合物.

本文以 $\mathrm{R}-\mathrm{Ga}-\mathrm{Mn}$ 三元体系为研究对象, $\mathrm{Ga}$ 提供 半导体性, $3 \mathrm{~d}$ 过渡族元素 $\mathrm{Mn}$ 和稀土元素 $\mathrm{Gd}$ 的共同 交换作用提供磁性. 通过磁性调制半导体元素的电 子输运性能.

\section{1 实验}

（i ）合金制备. 本实验所设计的材料结构式为 $\mathrm{GdGa}_{3-x} \mathrm{Mn}_{x}(x=0,0.1,0.2,0.3)$, 所选用的原料纯度均 高于 $99.9 \%$, 按化学式配比配制 $5 \mathrm{~g}$ 的试样, 由于稀 土和 $\mathrm{Mn}$ 元素具有挥发性, 对这些元素的质量给予了 适当的补偿：稀土为 $2 \%$ 3\%, $\mathrm{Mn}$ 为 10\% 15\%. 应用 非自耗真空电弧炉在高纯氩气保护下反复熔炼得到 成分均匀的铸锭; 然后对其进行退火处理 $\left(800^{\circ} \mathrm{C}\right.$, $36 \mathrm{~h}$ ), 以消除组织的残余应力, 得到成分均匀的相 结构; 最后将退火后的样品进行打磨、抛光, 并采用 日本岛津 $\mathrm{X}$ 射线苂光光谱仪(XRF, 1800 型)测定其 成分.

(ii) 晶体结构分析. 采用日本理学 $\mathrm{X}$ 射线衍射 仪(XRD, D/max 2500 立式), 扫描范围 $20^{\circ} \sim 80^{\circ}$, 管电 压 $40 \mathrm{kV}$, 管电流 $200 \mathrm{~mA}$, 步进扫描 $0.02 \%$ s. 用 TREOR 程序指标化, 用 Rietveld 峰形拟合修正的方 法进行结构精修.

(iii) 磁性能分析. 采用振动样品磁强计(VSM) 测量了试样磁化曲线( $(\mathrm{M}-\mathrm{H})$, 并对其在 0 1.5 $\mathrm{T}$ 磁场 下的 $\mathrm{M}-\mathrm{H}$ 曲线进行拟合, 得到各参数数值.

\section{2 结果与讨论}

\section{1 元素成分分析}

应用 XRF 测定 $\mathrm{GdGa}_{3-x} \mathrm{Mn}_{x}$ 样品中各元素的质量 百分比. 测试结果显示样品经熔炼和热处理后, 样品 中各元素质量百分比与设计的结构式 $\mathrm{GdGa}_{3-x} \mathrm{Mn}_{x}$ $(x=0,0.1,0.2,0.3)$ 接近. 表 1 给出标样 $\mathrm{GdGa}_{2.9} \mathrm{Mn}_{0.1}$ 的元素测试结果, 可知稀土元素 $\mathrm{Gd}$ 和过渡族元素
表 $1 \mathrm{GdGa}_{2.9} \mathrm{Mn}_{0.1}$ 各元素质量百分比

\begin{tabular}{cccc}
\hline 元素 & $\mathrm{Gd}(\%)$ & $\mathrm{Ga}(\%)$ & $\mathrm{Mn}(\%)$ \\
\hline 设计成分 & 43.0900 & 55.4067 & 1.5067 \\
实验成分 & 43.2676 & 55.0853 & 1.6567 \\
测量成分 & 42.1260 & 56.3865 & 1.4876 \\
\hline
\end{tabular}

$\mathrm{Mn}$ 均具有挥发性, 故配料时给了相应的补偿.

\section{2 物相检索与指标化}

$\mathrm{GdGa}_{3-x} \mathrm{Mn}_{x}(x=0,0.1,0.2,0.3)$ 样品的 XRD 图谱 如图 1 所示. $\mathrm{GdGa}_{3}$ 具有单相结构, 随着 $\mathrm{Mn}$ 含量的增 加, 其余 3 个样品出现少量未知杂相.

应用 TREOR 指标化方法对于 $\mathrm{GdGa}_{3-x} \mathrm{Mn}_{x}$ 主相 的晶面指数进行指标化, 并测定了其点阵参数. 表 2 列出其中的一个标样 $\mathrm{GdGa}_{2.7} \mathrm{Mn}_{0.3}$ 的指标化结果.

根据指标化结果, $\mathrm{GdGa}_{2.7} \mathrm{Mn}_{0.3}$ 主相具有六方结 构. 点阵常数为 $a=4.243(5) \AA, c=4.153(7) \AA$, 晶胞体 积 $V$ 为 74.766(6) $\AA^{3}$. 品质因子和可信度因子分别为 $M(19)=261, F(19)=146$ ，表明指标化结果可信.

其他 $\mathrm{GdGa}_{3-x} \mathrm{Mn}_{x}$ 合金化合物的主相点阵常数测 定结果, 如表 3 所示. 可以看出, 随着 $\mathrm{Mn}$ 含量的增 加, 点阵常数 $a, c$ 和晶胞体积 $V$ 都呈现出单调性的下 降. 其原因在于原子半径的差异. $\mathrm{Gd}$ 的原子半径为 $1.79 \AA, \mathrm{Ga}$ 的半径为 $1.23 \AA, \mathrm{Mn}$ 的原子半径为 $1.12 \AA$. 由于 $\mathrm{Mn}$ 的原子半径较 $\mathrm{Ga}$ 的原子半径小, 以 $\mathrm{Mn}$ 取 代 $\mathrm{Ga}$ 将导致 $a, c$ 都有所减小, 同时导致晶胞体积的 下降.

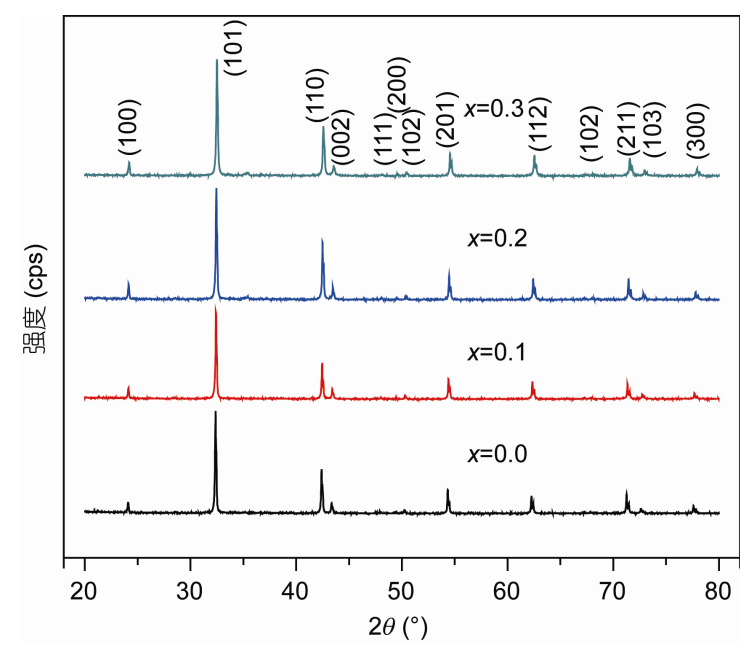

图 1 (网络版彩色) $\mathrm{GdGa}_{3-x} \mathrm{Mn}_{x}$ 化合物 XRD 图谱 
表 $2 \mathbf{G d G a}_{2.7} \mathbf{M n}_{\mathbf{0 . 3}}$ 主相衍射峰的指标化结果 ${ }^{\text {a) }}$

\begin{tabular}{|c|c|c|c|c|}
\hline$h k l$ & $\sin ^{2} \theta_{\mathrm{obs}}$ & $\sin ^{2} \theta_{\text {cal }}$ & $2 \theta_{\mathrm{obs}}$ & $2 \theta_{\mathrm{cal}}$ \\
\hline 0001 & 0.034281 & 0.034393 & 21.34 & 21.375 \\
\hline 100 & 0.043904 & 0.043924 & 24.19 & 24.195 \\
\hline 101 & 0.078304 & 0.078316 & 32.50 & 32.503 \\
\hline 110 & 0.131715 & 0.131771 & 42.56 & 42.569 \\
\hline 002 & 0.137553 & 0.137570 & 43.54 & 43.543 \\
\hline 111 & 0.166149 & 0.166163 & 48.11 & 48.112 \\
\hline 200 & 0.175674 & 0.175694 & 49.56 & 49.563 \\
\hline 102 & 0.181490 & 0.181494 & 50.43 & 50.431 \\
\hline 201 & 0.210359 & 0.210087 & 54.60 & 54.562 \\
\hline 112 & 0.269358 & 0.269341 & 62.53 & 62.528 \\
\hline 210 & 0.307450 & 0.307465 & 67.35 & 67.352 \\
\hline 202 & 0.313182 & 0.313264 & 68.06 & 68.070 \\
\hline 211 & 0.341844 & 0.341857 & 71.56 & 71.562 \\
\hline 103 & 0.353480 & 0.353456 & 72.96 & 72.957 \\
\hline 300 & 0.395276 & 0.395312 & 77.91 & 77.914 \\
\hline
\end{tabular}

a) $h k l$ 为晶面指数

表 $3 \operatorname{GdGa}_{3-x} \mathrm{Mn}_{x}(x=0,0.1,0.2,0.3)$ 合金化合物主相晶格 常数

\begin{tabular}{cccc}
\hline 点阵参数 & $a(\AA)$ & $c(\AA)$ & $V\left(\AA^{3}\right)$ \\
\hline$x=0.0$ & $4.258(4)$ & $4.170(4)$ & $75.604(4)$ \\
$x=0.1$ & $4.255(5)$ & $4.164(8)$ & $75.389(3)$ \\
$x=0.2$ & $4.249(4)$ & $4.159(5)$ & $75.086(5)$ \\
$x=0.3$ & $4.243(5)$ & $4.153(7)$ & $74.766(6)$ \\
\hline
\end{tabular}

对于样品中少量的杂相, 在目前 $\mathrm{X}$ 射线衍射数 据库(ICCD)中并未查询到与之相匹配的相, 其中包 括它们与 $\mathrm{C}, \mathrm{N}$ 和 $\mathrm{O}$ 形成的二元和三元化合物. 应用 最小二乘法对杂相的衍射峰进行指标化, 并测定其 点阵参数, 结果见表 $\mathrm{S} 1$. 根据指标化结果, $\mathrm{GdGa}_{2.7}$ $\mathrm{Mn}_{0.3}$ 中未知相具有正交结构, 点阵常数分别为 $a=$ 6.882(5) $\AA, b=13.286(5) \AA, c=7.002(7) \AA$, 晶胞体积 $V=640.360(0) \AA^{3}$. 品质因子和可信度因子分别为 $M(12)=9, \quad F(12)=4$. 此相的最强双峰 $\left(35.121^{\circ}\right.$ 和 $\left.35.417^{\circ}\right)$ 的晶面指数分别为(132)和(231).

\subsection{Rietveld 结构精修}

应用 Rietveld 结果精修方法测定 $\mathrm{GdGa}_{3-x} \mathrm{Mn}_{x}$ 的 晶体结构. 图 2 为其中 1 个标样 $\mathrm{GdGa}_{2.7} \mathrm{Mn}_{0.3}$ 的精修 XRD 图谱.

表 4 分别给出 $\mathrm{GdGa}_{3-x} \mathrm{Mn}_{x}(x=0,0.1,0.2,0.3)$ 体系
样品的 Rietveld 精修结构参数(原子占位、空间群以 及可信度因子). 稀土原子 $\mathrm{Gd}$ 占据 $1 \mathrm{a}$ 晶位; $\mathrm{Ga}$ 原子 有 2 种不同的晶位, 分别是 $\mathrm{Ga}^{\mathrm{I}}$ 原子占据 $2 \mathrm{~d}$ 晶位, $\mathrm{Ga}^{\mathrm{II}}$ 占据 $2 \mathrm{e}$ 晶位; $\mathrm{Mn}$ 原子部分替代 $\mathrm{Ga}$ 原子, 占据 $2 \mathrm{e}$ 晶 位. $\mathrm{GdGa}_{3-x} \mathrm{Mn}_{x}$ 的晶体结构, 如图 3 所示. $2 \mathrm{e}$ 晶位出 现( $\mathrm{Mn}, \mathrm{Ga})-(\mathrm{Mn}, \mathrm{Ga})$ 原子对, 与 $1 \mathrm{a}$ 晶位密切相关, 原 子对的中点, 即为 $1 \mathrm{a}$ 晶位的位置. $\mathrm{GdGa}_{3-x} \mathrm{Mn}_{x}$ 的结构 精修结果显示, 1a 晶位存在空位, 而 $2 \mathrm{e}$ 晶位的 $(\mathrm{Mn}$, $\mathrm{Ga})$ 原子含量很少, 空位较多. 其产生机理, 可以看 作是 $(\mathrm{Mn}, \mathrm{Ga})-(\mathrm{Mn}, \mathrm{Ga})$ 原子对部分替代 $1 \mathrm{a}$ 晶位的 $\mathrm{Gd}$ 所导致. 结构式可以写作 $\mathrm{Gd}_{1-r} \mathrm{Ga}_{2}(\mathrm{Ga}, \mathrm{Mn})_{2 r}$. 当 $r=$ 0.2 时, 结构式可以约化成 $\mathrm{Gd}(\mathrm{Ga}, \mathrm{Mn})_{3} \quad 1: 3$ 型结构. 这说明 $\mathrm{Gd}(\mathrm{Ga}, \mathrm{Mn})_{3} 1: 3$ 型化合物的形成是源于 $2 \mathrm{e}$ 晶 位 $\mathrm{Ga}$ 原子部分替代稀土原子所导致的无序结构相变.

\section{4 磁性能}

应用 VSM 测量了 $\mathrm{GdGa}_{3-} \mathrm{Mn}_{x}$ 样品在室温下的 磁化曲线, 如图 4 所示. 结果显示, 当 $x=0$ 时, 曲线 几乎为线性, 化合物为顺磁结构; 当 $0<x \leqslant 0.3$ 时, 化 合物为弱铁磁性和顺磁性的混合相; 当 $x=0.2$ 时, $\mathrm{Mn}$ 掺杂达到饱和, 此时得到的化合物磁性最强.

由于二元稀土化合物 $\mathrm{GdGa}_{3}$ 具有顺磁性, 故应 用顺磁性理论公式对其在 $0 \sim 1.5 \mathrm{~T}$ 磁场下的 M-H 曲线 进行拟合. 应用的理论模型见下式:

$$
M=\chi_{\mathrm{p}} H ，
$$

式中, $M$ 是磁化强度, $H$ 是磁场强度; $\chi_{\mathrm{p}}$ 是顺磁磁 化率.

由于 $\mathrm{GdGa}_{2.9} \mathrm{Mn}_{0.1}, \mathrm{GdGa}_{2.8} \mathrm{Mn}_{0.2}, \mathrm{GdGa}_{2.7} \mathrm{Mn}_{0.3}$ 三

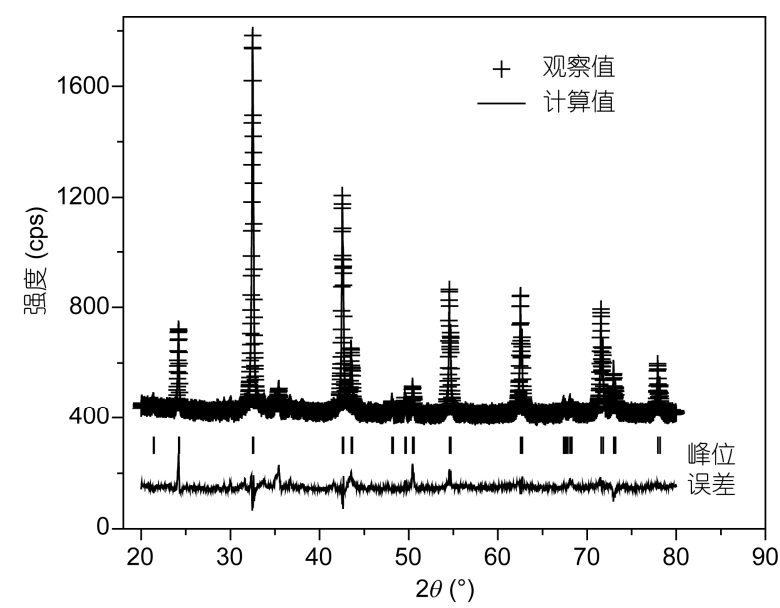

图 $2 \mathrm{GdGa}_{2.7} \mathrm{Mn}_{0.3}$ 合金的 Rietveld 结构精修图谱 
表 $4 \mathrm{GdGa}_{3-x} \mathrm{Mn}_{x}(x=0,0.1,0.2,0.3)$ 化合物 Rietveld 精修结构参数

\begin{tabular}{lcccc}
\hline \multicolumn{1}{c}{ 样品 } & $\mathrm{GdGa}_{3}$ & $\mathrm{GdGa}_{2.9} \mathrm{Mn}_{0.1}$ & $\mathrm{GdGa}_{2.8} \mathrm{Mn}_{0.2}$ & $\mathrm{GdGa}_{2.7} \mathrm{Mn}_{0.3}$ \\
\hline 空间群 & $P 6 / m m m$ & $P 6 / m m m$ & $P 6 / m m m$ \\
$a$ & $4.257(4)$ & $P 6 / m m m$ & $4.248(4)$ & $4.242(5)$ \\
$c$ & $4.168(4)$ & $4.253(5)$ & $4.157(5)$ & $0.151(7)$ \\
Gd-1a & 0 & $4.162(8)$ & 0 & 0 \\
& 0 & 0 & 0 & 0 \\
$N$ & 0 & 0 & 0 & $0.800(0)$ \\
$\mathrm{Ga}-2 \mathrm{~d}$ & $0.800(0)$ & 0 & $0.800(0)$ & $1 / 3$ \\
& $1 / 3$ & $0.800(0)$ & $1 / 3$ & $2 / 3$ \\
$N$ & $2 / 3$ & $1 / 3$ & $2 / 3$ & $1 / 2$ \\
$\mathrm{Ga} / \mathrm{Mn}$-2e & $1 / 2$ & $2 / 3$ & $1 / 2$ & $2.000(0)$ \\
& $2.000(0)$ & $1 / 2$ & $2.000(0)$ & 0 \\
$N$ & 0 & $2.000(0)$ & 0 & 0 \\
布拉格 $R$ 因子 $R_{\mathrm{B}}$ & 0 & 0 & 0 & $0.263(5)$ \\
结构 $R$ 因子 $R_{\mathrm{F}}$ & $0.117(4)$ & 0 & $0.185(6)$ & $0.416(9)$ \\
\hline
\end{tabular}

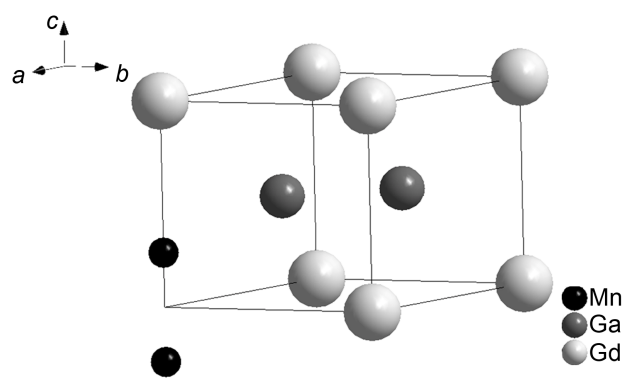

图 $3 \mathrm{GdGa}_{2.7} \mathrm{Mn}_{0.3}$ 的晶体结构图

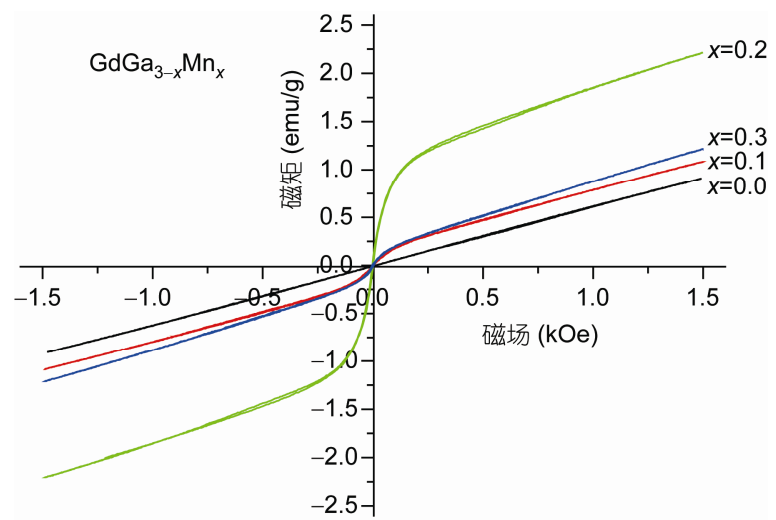

图 4 (网络版彩色) $\mathbf{G d G a}_{3-x} \mathrm{Mn}_{x}$ 化合物室温磁化曲线

种稀土化合物存在混合磁性，因此对其在 $0 \sim 1.5 \mathrm{~T}$ 磁 场下的磁化曲线采用趋近饱和定律进行拟合，拟合
公式为 ${ }^{[13]}$

$$
M=M_{\mathrm{s}}\left(1-a / H-b / H^{2}-c / H^{3}\right)+\chi_{\mathrm{p}} H,
$$

式中, $M_{\mathrm{s}}$ 代表饱和磁化强度, $a, b, c$ 分别是与外场及 其平方项和立方项有关的参数. 拟合曲线如图 5 所示, 图中圆圈所示为磁化实验数据, 实线为拟合曲线, 从 图中可见拟合结果均良好, 表 S2 列出了拟合参数.

图 6 所示为拟合结果中 $a, b, c$ 和换算后 $\chi_{\mathrm{p}}$ 和 $M_{\mathrm{s}}$ 各参数随 $\mathrm{Mn}$ 掺杂含量的对数变化曲线. 可见, 随着 $\mathrm{Mn}$ 含量的增多, $a, b, c$ 各参数均先减小后增大, 在 $x=0.2$ 时达到最小值; 然而, $\chi_{\mathrm{p}}$ 与 $M_{\mathrm{s}}$ 均表现出先增大 后减小的趋势, 在 $x=0.2$ 时达到最大值, 说明 $\mathrm{Mn}$ 的 掺杂有利于合金化合物中铁磁性含量的增加, 并在 掺杂量 $x=0.2$ 时达到最大值. 由于该系列掺 $\mathrm{Mn}$ 化合 物在室温下体现出部分铁磁性, 有进一步优化成全 部铁磁性的可能.

室温的磁化过程揭示, $\mathrm{GdGa}_{3-x} \mathrm{Mn}_{x}$ 呈现出铁磁 性和顺磁性共存的混合磁性特征. 顺磁性可能来源 于没有被 $\mathrm{Mn}$ 替代的 $\mathrm{GdGa}_{3}$ 团簇, 其顺磁磁化率在 $6.19 \times 10^{-3} \sim 7.87 \times 10^{-3}$; 铁磁性的形成则可能是由磁性 元素 $\mathrm{Mn}$ 部分替代 $\mathrm{Ga}$ 所导致. 化合物的磁矩与 $\mathrm{Mn}$ 的含量有关, 在 0.0376(3) 0.3541(2)变化, 这可能是 由于 $\mathrm{Mn}$ 原子间的间距变化造成的，当 $\mathrm{Mn}$ 原子间距 

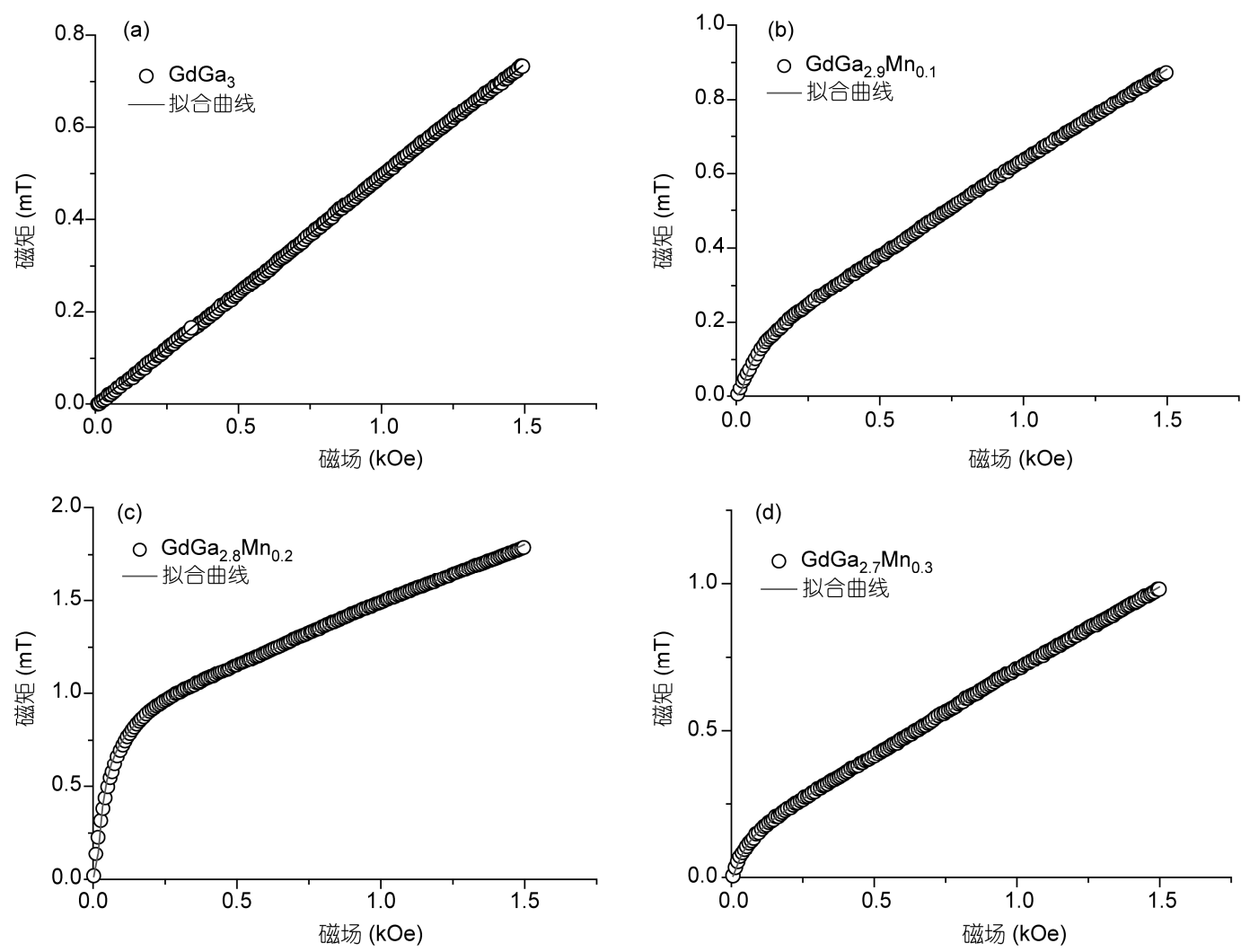

图 $5 \mathrm{GdGa}_{3-x} \mathrm{Mn}_{x}$ 化合物在 $\mathbf{0 ~ 1 . 5} \mathrm{T}$ 外场下的磁化拟合曲线

(a) $\mathrm{GdGa}_{3}$; (b) $\mathrm{GdGa}_{2.9} \mathrm{Mn}_{0.1}$; (c) $\mathrm{GdGa}_{2.8} \mathrm{Mn}_{0.2}$; (d) $\mathrm{GdGa}_{2.7} \mathrm{Mn}_{0.3}$

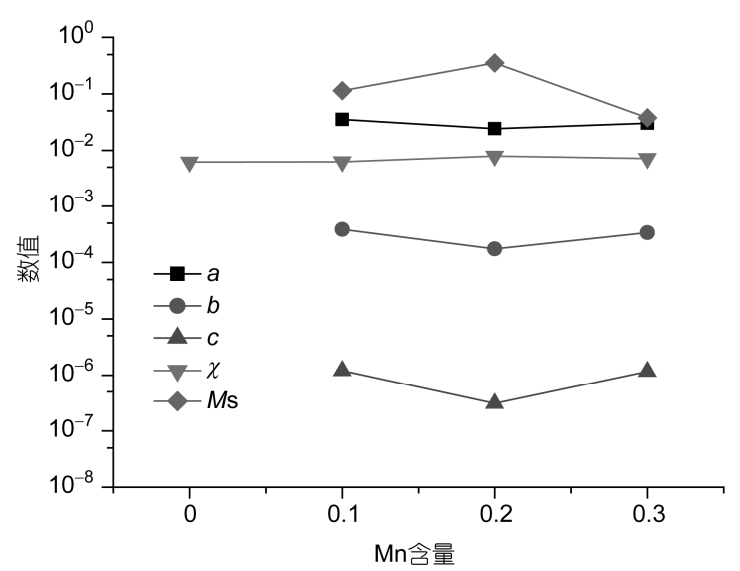

图 $6 \mathrm{GdGa}_{3-x} \mathrm{Mn}_{x}(x=0,0.1,0.2,0.3)$ 化合物磁化曲线中拟合 参数随 Mn 含量的变化曲线

较大时, $\mathrm{Mn}$ 原子间呈平行排列, 随着 $\mathrm{Mn}$ 掺杂的增 多, $\mathrm{Mn}$ 原子间距逐渐减小, 当 $x=0.2$ 时, 磁矩达到最 大值, $\mathrm{Mn}$ 原子间出现部分反平行排列, 从而造成磁 矩的降低.

根据拟合结果发现, $a$ 参数较大, $c$ 参数很小, 说
明铁磁性部分主要来自磁场的一次相系数 $a$; 随着铁 磁性 $\mathrm{Mn}$ 含量的增加, $a$ 先增大再减小. 由此可见, $\mathrm{Mn}$ 的掺杂对 $\mathrm{GdGa}_{3-x} \mathrm{Mn}_{x}$ 的磁性能产生了较大影响.

\section{3 结论}

本文用电弧熔炼法制备了 $\mathrm{GdGa}_{3-x} \mathrm{Mn}_{x}(x=0,0.1$, $0.2,0.3)$ 系列合金. $\mathrm{GdGa}_{3-x} \mathrm{Mn}_{x}(x=0,0.1,0.2,0.3)$ 中主 相具有六方结构, 空间群为 $P 6 / \mathrm{mmm}$, 点阵常数 $a=4.242(5) \sim 4.257$ (4) $\AA, c=4.151(7) \sim 4.168(4) \AA . \mathrm{Gd}$ 原 子占据 $1 \mathrm{a}(0,0,0)$ 晶位, $\mathrm{Ga}$ 原子分别占据 $2 \mathrm{~d}(1 / 3,2 / 3$, $1 / 2)$ 和 $2 \mathrm{e}(0,0, \mathrm{z})$ 晶位, $\mathrm{Mn}$ 原子部分替代 $2 \mathrm{e}$ 晶位的 $\mathrm{Ga}$ 原子. 随着 $\mathrm{Mn}$ 含量的增加, 主相的点阵常数 $a$ 和 $c$, 晶胞体积 $V$ 都出现单调性的下降. 这是由于尺寸因 素所致, 由于 $\mathrm{Mn}$ 的原子半径较 $\mathrm{Ga}$ 的原子半径小, $\mathrm{Mn}$ 替代 $\mathrm{Ga}$ 导致晶格畸变, $a, c$ 都有所减小, 同时晶 胞体积下降. $\mathrm{GdGa}_{3-x} \mathrm{Mn}_{x}$ 的磁测量结果表明, 当 $x=0$ 时, 为顺磁结构; 当 $0<x \leqslant 0.3$ 时, 为弱铁磁性和顺磁 性的混合, 磁化过程符合趋近饱和定律. 磁化曲线拟 
合参数 $a, b, c$ 随着 $\mathrm{Mn}$ 含量的增加呈现先减后增的

趋势, $\chi_{\mathrm{p}}$ 与 $M_{\mathrm{s}}$ 则是先增后减, 并均在 $x=0.2$ 时达到极
值. 由此可见, $\mathrm{Mn}$ 的掺杂对 $\mathrm{GdGa}_{3-x} \mathrm{Mn}_{x}$ 的磁性能产 生了较大影响.

\title{
参考文献
}

1 Kletowski Z, Fabrowski R, Slawiński P, et al. Resistance of some $\mathrm{REMe}_{3}$ compounds ( $\mathrm{RE}=\mathrm{La}$ and $\mathrm{Lu}, \mathrm{Me}=\mathrm{Sn}, \mathrm{Pb}, \mathrm{In}$, and Ga). J Magn Magn Mater, 1997, 166: 361-364

2 Kletowski Z. Crystal growth and some properties of $\mathrm{REMe}_{3}$ compounds (RE=rare earth, $\mathrm{Me}=\mathrm{Sn}, \mathrm{Pb}$, Ga, and In). In: Rudi C, Marcel A, Marek P, et al., eds. Supermaterials. Dordrecht: Kluwer Academic Publishers, 2000. 163-172

3 Kletowski Z, Czopnik A, Tal A, et al. High magnetic field properties of GdIn 3 . Phys Rev B: Condens Matter, 2000, 281: 163-164

4 Palenzona A, Clrafici S. The Ga-Gd (Gallium-Gadolinium) system. Bull Alloy Phase Diagrams, 1990, 11: 67-72

5 Barandiaran J, Gignoux D, Rodriguez-Fernandez J. Magnetic properties and magnetic structure of hexagonal $\mathrm{GdGa}_{2} \mathrm{and}_{\mathrm{GdCu}_{5}}$ compounds. Phys Rev B: Condens Matter, 1989, 154: 293-299

6 Ohara S, Sakamoto I, Aoki Y, et al. Magnetic properties and de Haas-van Alphen effect of GdGa 2 . Phys Rev B: Condens Matter, 1996, 223: $379-381$

7 Bouvier M, Lethuillier P, Schmitt D. Specific heat in some gadolinium compounds. I. Experimental. Phys Rev B: Condens Matter, 1991, 43: $13137-13144$

8 Mulder F, Thiel R, Buschow K. ${ }^{155} \mathrm{Gd}$ Mössbauer effect and magnetic properties of $\mathrm{GdNi}_{2} \mathrm{Sb}_{2}, \mathrm{GdCu}_{2} \mathrm{Sb}_{2}$ and $\mathrm{GdAl}_{2} \mathrm{Ga}_{2}$. J Alloys Compd, 1994, 210: 335-338

9 Hamasaki T, Deguchi H. Magnetic phase diagram of $\mathrm{GdGa}_{1.75} \mathrm{Al}_{0.25}$. Phys Rev B: Condens Matter, 2003, 329: 645-646

10 Tung L, Thuy N, Brommer P, et al. Magnetic properties of the pseudo-binary $\mathrm{GdGa}_{2-x} \mathrm{Al}_{x}$ compounds. Phys Rev B: Condens Matter, 1999 , 266: $209-216$

11 Morin $\mathrm{P}$, Giraud M, Regnault $\mathrm{P}$, et al. Crystalline electric field and magnetic structure of $\mathrm{RGa}_{3}$ compounds within the AuCu $\mathrm{u}_{3}-\mathrm{type}$ structure. J Magn Magn Mater, 1987, 66: 345-350

12 Luo J, Liang J K, Guo Y Q, et al. Effects of the doping element on crystal structure and magnetic properties of $\operatorname{Sm}(\mathrm{Co}, \mathrm{M})_{7}$ compounds $(\mathrm{M}=\mathrm{Si}, \mathrm{Cu}, \mathrm{Ti}, \mathrm{Zr}$, and $\mathrm{Hf})$. Intermetallics, 2005, 13: 710-716

13 钟文定. 铁磁学(中册). 北京: 科学出版社, 1992.277

\section{Structure and magnetic properties of novel $\mathrm{GdGa}_{3-x} \mathrm{Mn}_{x}$ intermetallic compounds}

\author{
HE Qiang, LIU HanYuan \& GUO YongQuan \\ School of Energy Power and Mechanical Engineering, North China Electric Power University, Beijing 102206, China
}

$\mathrm{GdGa}_{3-x} \mathrm{Mn}_{x}$ intermetallic compounds were prepared using an Arc-melting technology under an atmosphere of Argon gas. The phase structures were systemically investigated with powder X-ray diffractometer. The planar indices and lattice parameters were determined with TREOR program and a least square method. A Rietveld structural refinement technology was employed to determine the crystal structures of $\mathrm{GdGa}_{3-x} \mathrm{Mn}_{x}(x=0,0.1,0.2,0.3)$. It shows that the main phase in $\mathrm{GdGa}_{3-x} \mathrm{Mn}_{x}$ crystallizes in a hexagonal structure with space group of $P 6 / \mathrm{mmm}$. Gd atoms occupy the 1a crystal position with $20 \%$ vacancy. Ga atoms are at $2 \mathrm{~d}$ and $2 \mathrm{e}$ site. Mn atoms partly substitute for $\mathrm{Ga}$ at 2e position. The field dependence of magnetization of $\mathrm{GdGa}_{3-x} \mathrm{Mn}_{x}$ was measured using a vibrating sample magnetometer. The contributions to magnetic moments originate from the doped $\mathrm{Mn}$ atoms. With increasing Mn content in $\mathrm{GdGa}_{3-x} \mathrm{Mn}_{x}$, the magnetic phases exhibit a transition from paramagnetic to mixture characters of weak ferromagnetic and paramagnetic properties. According to the fitting results of M-H curve, with increasing Mn content, the parameters of $a, b$ and $c$ first decrease and then increase, but the susceptibility $\chi_{\mathrm{P}}$ and saturation moment $M \mathrm{~s}$ first increase and then decrease, and the maximum values for all the parameters present at $x=0.2$. It implies that $\mathrm{Mn}$ atoms play an important role in magnetic properties of $\mathrm{GdGa}_{3-x} \mathrm{Mn}_{x}$ compounds.

\section{$\mathrm{GdGa}_{3-x} \mathrm{Mn}_{x}$, Rietveld refinement, magnetic}

doi: 10.1360/N972013-00033 


\section{补充材料}

表 S1 $\mathrm{GdGa}_{2.7} \mathrm{Mn}_{0.3}$ 中未知相衍射峰的指标化结果

表 S2 $\mathrm{GdGa}_{3-x} \mathrm{Mn}_{x}(x=0,0.1,0.2,0.3)$ 化合物磁化曲线的拟合参数

本文的以上补充材料见网络版 csb.scichina.com. 补充材料为作者提供的原始数据, 作者对其学术质量和内容负责. 
表 S1 $\mathrm{GdGa}_{2.7} \mathrm{Mn}_{0.3}$ 中未知相衍射峰的指标化结果

\begin{tabular}{|c|c|c|c|c|}
\hline hkl & $\sin ^{2} \theta_{\mathrm{obs}}$ & $\sin ^{2} \theta_{\text {cal }}$ & $2 \theta_{\mathrm{obs}}$ & $2 \theta_{\mathrm{cal}}$ \\
\hline 102 & 0.061093 & 0.060924 & 28.620 & 28.580 \\
\hline 112 & 0.064068 & 0.064285 & 29.324 & 29.375 \\
\hline 050 & 0.084188 & 0.084026 & 33.734 & 33.701 \\
\hline 132 & 0.091031 & 0.091173 & 35.121 & 35.149 \\
\hline 231 & 0.092522 & 0.092452 & 35.417 & 35.403 \\
\hline 042 & 0.102354 & 0.102175 & 37.317 & 37.283 \\
\hline 320 & 0.126050 & 0.126177 & 41.591 & 41.613 \\
\hline 162 & 0.181787 & 0.181922 & 50.474 & 50.494 \\
\hline 252 & 0.182433 & 0.182527 & 50.570 & 50.584 \\
\hline 053 & 0.192988 & 0.192921 & 52.119 & 52.109 \\
\hline 500 & 0.313208 & 0.313146 & 68.063 & 68.056 \\
\hline 105 & 0.315023 & 0.315012 & 68.287 & 68.286 \\
\hline
\end{tabular}

表 S2 $\mathrm{GdGa}_{3-x} \mathrm{Mn}_{x}(x=0,0.1,0.2,0.3)$ 化合物磁化曲线的拟合参数

\begin{tabular}{|c|c|c|c|c|c|}
\hline & & $\mathrm{GdGa}_{3}$ & $\mathrm{GdGa}_{2.9} \mathrm{Mn}_{0.1}$ & $\mathrm{GdGa}_{2.8} \mathrm{Mn}_{0.2}$ & $\mathrm{GdGa}_{2.7} \mathrm{Mn}_{0.3}$ \\
\hline 平均残差平方 & & $1.29126 \times 10^{-5}$ & $1.21285 \times 10^{-5}$ & $2.9691 \times 10^{-4}$ & $1.09406 \times 10^{-5}$ \\
\hline 修正决定系数 & & 0.99972 & 0.99977 & 0.99766 & 0.99984 \\
\hline \multirow{5}{*}{ 拟合参数 } & $a$ & - & 0.03522 & 0.02408 & 0.03016 \\
\hline & $b$ & - & $-3.86857 \times 10^{-4}$ & $-1.7464 \times 10^{-4}$ & $-3.36971 \times 10^{-4}$ \\
\hline & $c$ & - & $1.19404 \times 10^{-6}$ & $3.12051 \times 10^{-7}$ & $1.159 \times 10^{-6}$ \\
\hline & $\chi_{p}$ & 0.49232 & 0.49591 & 0.62622 & 0.56822 \\
\hline & $M_{s}$ & - & 0.14135 & 0.87842 & 0.14062 \\
\hline \multirow{5}{*}{ 拟合参数的误差 } & $A$ & - & $4.70444 \times 10^{-4}$ & $2.71961 \times 10^{-4}$ & $5.1098 \times 10^{-4}$ \\
\hline & $B$ & - & $9.65766 \times 10^{-6}$ & $3.40122 \times 10^{-6}$ & $1.19354 \times 10^{-5}$ \\
\hline & $C$ & - & $3.90176 \times 10^{-8}$ & $7.29381 \times 10^{-9}$ & $5.65873 \times 10^{-8}$ \\
\hline & $\chi_{p}$ & $2.20408 \times 10^{-4}$ & $5.87663 \times 10^{-4}$ & 0.00267 & $5.67405 \times 10^{-4}$ \\
\hline & $M s$ & - & $6.23277 \times 10^{-4}$ & 0.00271 & $6.12494 \times 10^{-4}$ \\
\hline \multirow{2}{*}{$\begin{array}{l}\text { 磁化率和饱和磁矩 } \\
\text { 的计算结果 }\end{array}$} & $\chi_{p}$ & 0.00619 & 0.00623 & 0.00787 & 0.00714 \\
\hline & $M_{s}$ & - & 0.11443 & 0.35412 & 0.03763 \\
\hline
\end{tabular}

